\title{
DIAMOND AMPLIFIED PHOTOCATHODES ${ }^{*}$
}

\author{
John Smedley ${ }^{1}$, Ilan Ben-Zvi ${ }^{1}$, Jen Bohon ${ }^{2}$, Xiangyun Chang ${ }^{1}$, \\ Ranjan Grover ${ }^{1}$, Abdel Isakovic ${ }^{1}$, Triveni Rao ${ }^{1}$, and Qiong $\mathrm{Wu}^{3}$ \\ ${ }^{1}$ Brookhaven National Laboratory, Upton, NY 11973-5000 \\ ${ }^{2}$ Case Western Reserve University, Cleveland, OH 44106 \\ ${ }^{3}$ Indiana University, Bloomington, IN 47405
}

November, 2007

"This manuscript has been authored by Brookhaven Science Associates, LLC under Contract No. DE-AC02-98CH10886 with the U.S. Department of Energy. The United States Government retains, and the publisher, by accepting the article for publication, acknowledges, a world-wide license to publish or reproduce the published form of this manuscript, or allow others to do so, for the United States Government purposes. 


\section{DISCLAIMER}

This work was prepared as an account of work sponsored by an agency of the United States Government. Neither the United States Government nor any agency thereof, nor any of their employees, nor any of their contractors, subcontractors, or their employees, makes any warranty, express or implied, or assumes any legal liability or responsibility for the accuracy, completeness, or any third party's use or the results of such use of any information, apparatus, product, or process disclosed, or represents that its use would not infringe privately owned rights. Reference herein to any specific commercial product, process, or service by trade name, trademark, manufacturer, or otherwise, does not necessarily constitute or imply its endorsement, recommendation, or favoring by the United States Government or any agency thereof or its contractors or subcontractors. The views and opinions of authors expressed herein do not necessarily state or reflect those of the United States Government or any agency thereof. 


\title{
Diamond Amplified Photocathodes
}

\author{
John Smedley ${ }^{1}$, Ilan Ben-Zvi ${ }^{1}$, Jen Bohon ${ }^{2}$, Xiangyun Chang ${ }^{1}$, Ranjan Grover ${ }^{1}$, Abdel Isakovic ${ }^{1}$, \\ Triveni Rao ${ }^{1}$, and Qiong $\mathrm{Wu}^{3}$ \\ ${ }^{1}$ Brookhaven National Laboratory, Upton, NY, 11973 \\ ${ }^{2}$ Case Western Reserve University, Clevand, OH, 44106 \\ ${ }^{3}$ Indiana University, Bloomington, IN, 47405
}

\begin{abstract}
High-average-current linear electron accelerators require photoinjectors capable of delivering tens to hundreds of $\mathrm{mA}$ average current, with peak currents of hundreds of amps. Standard photocathodes face significant challenges in meeting these requirements, and often have short operational lifetimes in an accelerator environment. We report on recent progress toward development of secondary emission amplifiers for photocathodes, which are intended to increase the achievable average current while protecting the cathode from the accelerator.

The amplifier is a thin diamond wafer which converts energetic (few keV) primary electrons into hundreds of electron-hole pairs via secondary electron emission. The electrons drift through the diamond under an external bias and are emitted into vacuum via a hydrogenterminated surface with negative electron affinity (NEA). Secondary emission gain of over 200 has been achieved. Two methods of patterning diamond, laser ablation and reactive-ion etching (RIE), are being developed to produce the required geometry. A variety of diagnostic techniques, including FTIR, SEM and AFM, have been used to characterize the diamonds.
\end{abstract}

\section{INTRODUCTION}

Linear electron accelerators have long been capable of producing high average currents $(>1 \mathrm{~A})$ or low emittance/high brightness $(\varepsilon \sim 1 \mu \mathrm{m} \cdot \mathrm{rad})$. Many emerging applications of these accelerators, such as free-electron lasers, linac light sources and ion cooling [1,2] require a high quality beam and a high average current. Direct generation of high currents from photocathodes requires highly efficient cathodes and very powerful lasers. High quantum efficiency (QE) cathodes tend to have short operational lifetimes in accelerator environments, especially when operated at high current. High QE cathodes also tend to use cesium, which has the potential to adversely affect the performance of superconducting photoinjector cavities. In order to alleviate these problems, we are investigating the use of a secondary emitter that will amplify the primary current while acting as a barrier between the primary cathode and the cavity. Synthetic diamond has exhibited secondary electron yield (gain) exceeding 100 [3]. The electrical, mechanical and thermal properties of diamond also make it an ideal candidate for this application.

The field of electron transport in and emission from diamond has received considerable work in recent years. Electron emission has been directly measured in a reflection geometry by Yater $e t$ al. [4] using hydrogen as well as cesium termination to achieve NEA. This work also measured the energy spectrum of the emitted electrons, showing that the majority of the emitted electrons have energies near the vacuum level, with a FWHM energy spread of $0.54 \mathrm{eV}$ for hydrogenated, 100 oriented natural diamond. A gain (the ratio of secondary to primary electron current) of 60 
was observed for $3 \mathrm{keV}$ primary electron energy. Electron transport in diamond has been modeled by Watanabi et al. [5]. Their model shows that for bias fields of a few $\mathrm{MV} / \mathrm{m}$ in diamond, the electron velocity saturates (at $>10^{5} \mathrm{~m} / \mathrm{s}$ ), yet the electrons in the conduction band of diamond still have an average energy within $0.1 \mathrm{eV}$ of the conduction band minimum. These results are encouraging for a diamond amplified photocathode (DAP), as they suggest that significant current amplification is possible, and that the energy spread of the emitted electrons should be comparable to the energy spread of electrons from a standard photocathode $(0.1-1.0 \mathrm{eV})$.
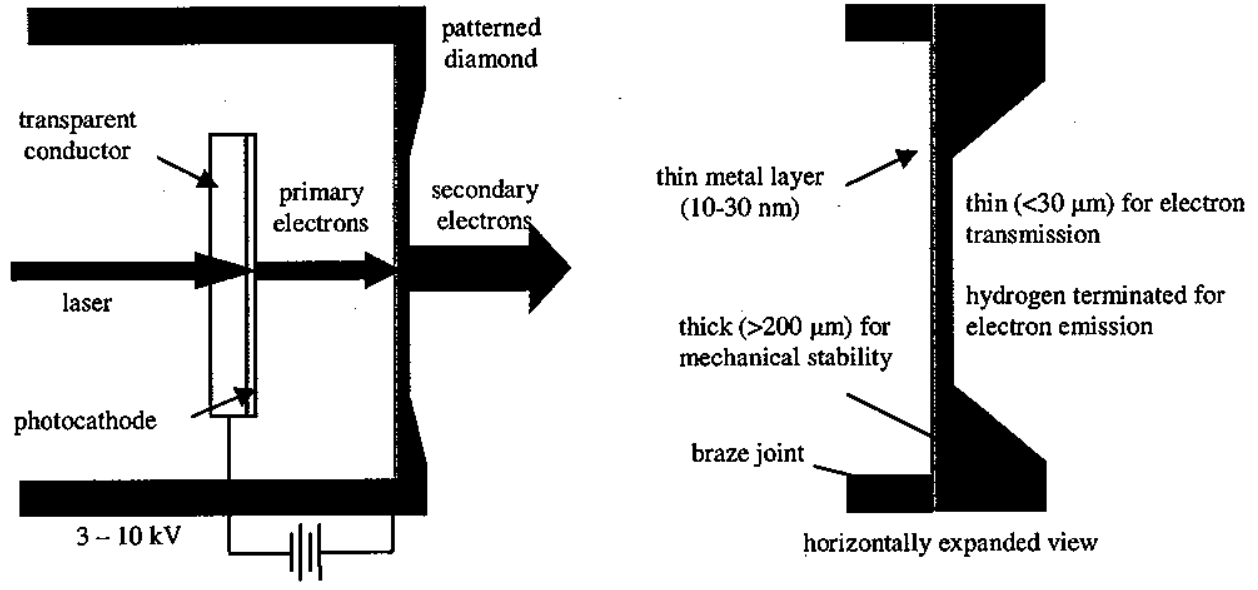

Figure 1. Diamond amplified cathode concept

A schematic representation of the DAP is shown in figure 1. A pulsed laser is used to create a primary electron bunch from a transmission-mode photocathode. The laser is shaped to produce the desired temporal and spatial profile of the electron bunch, typically a spatial and temporal flat top with a pulse duration of $\sim 10 \mathrm{ps,} \mathrm{although} \mathrm{the} \mathrm{bunch} \mathrm{properties} \mathrm{will} \mathrm{vary} \mathrm{with}$ application. A DC bias of several $\mathrm{kV}$ is used to accelerate the primary electrons, which pass through a thin metallization layer and into the diamond. The electrons lose energy in the diamond primarily via electron-electron scattering, creating additional electron-hole pairs. Within a fraction of a picosecond [6], the electrons have lost kinetic energy to a point that they can no longer produce further secondaries. The electrons then thermalize to the bottom of the conduction band via electron-phonon scattering. A bias is applied on the diamond, causing the electrons to drift toward the vacuum interface and the holes to drift toward the metal layer. This bias can in principal be DC or RF; this work will proceed assuming that the amplifier is being used in a RF photoinjector and that the electric field of the cavity is used to pull the electrons through the diamond. In this case, the electrons must drift through the diamond and be emitted within the positive half-cycle of the RF. This puts a limit on the maximum thickness of diamond that can be used. In a standard photoinjector, the accelerating field for the electrons in vacuum will be $>20 \mathrm{MV} / \mathrm{m}$, leading to a field of several $\mathrm{MV} / \mathrm{m}$ in diamond (the dielectric constant of diamond is 5.7). This is a good range to operate the amplifier, as the models show this field is sufficient to saturate the electron velocity but low enough to avoid significant increase in the energy spread of the emitted electrons. The surface of the diamond will be hydrogen terminated to achieve NEA, ideally allowing the electrons to be emitted into vacuum.

\section{EXPERIMENT}

The development of the diamond amplifier presents a series of technical challenges both in the choice of materials and the preparation techniques. The ideal device is comprised of a thin, 
pure diamond metallized on one side and hydrogenated on the other. It must be in good thermal contact with the cavity and good electrical contact with a current resupply source, but the amplifier must remain electrically isolated from the photocathode. Current progress in addressing these challenges is reported below. A variety of characterization techniques have been employed to evaluate success in obtaining the desired properties.

\section{Diamond properties}

The choice of diamond substrate for the amplifier requires an understanding of the role of imperfections within the diamond. The interaction of electrons with areas of impurity or crystalline grain boundaries can result in trapping of those electrons within the diamond bulk. The properties of both natural and several grades of CVD grown synthetic diamond have been characterized via Fourier transform infrared (FTRR) and Raman/photoluminescence spectroscopy (R/PL). FTIR spectra of a natural (ND, purple), a single crystal detector grade (DD, magenta) and a polycrystalline electronic grade diamond (ED, red) are shown in figure 2 (left).
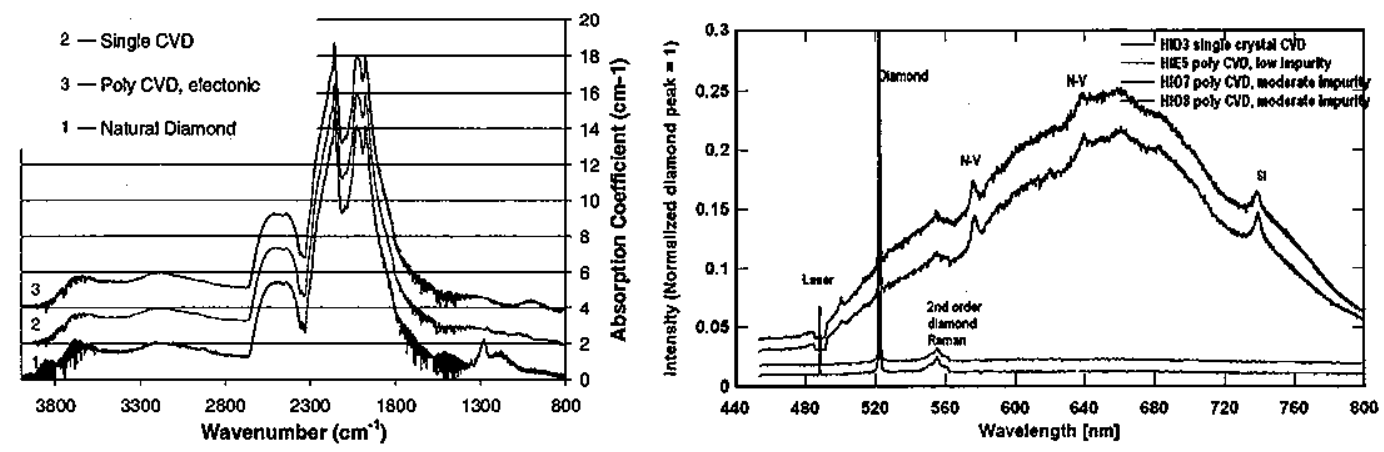

Figure 2. Left: IR absorption spectra; Right: Raman/photoluminescence spectra The structure of these spectra is characteristic of the diamond lattice, with the exception of the peak at $1282 \mathrm{~cm}^{-1}$ in the ND spectrum, which corresponds to $\sim 30 \mathrm{ppm}$ of type IaA nitrogen aggregate impurity (typical for ND). The synthetic diamonds have no observable peaks in the nitrogen region, $\left(1100 \mathrm{~cm}^{-1}\right.$ and $\left.1300 \mathrm{~cm}^{-1}\right)$, implying that the nitrogen impurity levels are $<1 \mathrm{ppm}$. The R/PL spectra (Fig. 2, right) are consistent with negligible nitrogen levels in the ED (green) and DD (red) samples, but clearly indicate significant nitrogen and silicon impurities in the optical grade (OD, black and blue) diamonds. The OD spectra also exhibit significant photoluminescence, likely due not only to impurities, but also to the abundance of randomly oriented crystalline grain boundaries (10 $\mu \mathrm{m}$ grain size), in contrast to the SD or large grain $(100 \mu \mathrm{m})$ ED samples. It is clear from these results that OD samples are not acceptable candidates for further development. DD samples would be ideal, but large enough crystals are not commercially available. High-quality ND samples are also suitable, but are expensive and inherently not reproducible. ED samples can be grown large enough and are reasonably priced, but may produce non-uniform emission, as the electron affinity of each grain will differ: Diffraction studies indicate that the major crystalline orientations present in the ED samples are 110, 211 and 331 (ND: 110, DD: 100). Techniques to enhance a particular crystalline orientation (preferably 111) within the ED samples are currently under development.

\section{Electron transport in diamond}

Demonstration of the production and transport of secondary electrons in diamond is central to the development of the amplifier. The system used to measure secondary electron yield (gain) in diamond has been previously described [7]. For the measurements shown in figure 3, 
the electron beam had a spot size of $4 \mathrm{~mm}^{2}$, a primary current of $300 \pm 40 \mathrm{nA}$ and a pulse duration of $10 \mu \mathrm{s}$. The detector grade diamond sample $(4 \mathrm{~mm} \times 4 \mathrm{~mm} \times 0.3 \mathrm{~mm})$ was metallized via sputtering on both sides with $15 \mathrm{~nm}$ Ti and $25 \mathrm{~nm}$ Pt. No thermal anneal was done on this sample.

The gain (Fig. 3, left) saturates above $0.5 \mathrm{MV} / \mathrm{m}$, indicating that for this current density. and diamond purity, recombination of electrons and holes is significant below this value. Figure 3 (right) displays the saturated gain as a function of the primary energy. The $\mathrm{x}$ intercept of this plot gives the energy the primary electrons lose in the metal coating of the diamond, and the slope gives the average electron energy required to produce an electron-hole pair $(20 \mathrm{eV})$. This value is somewhat higher than that observed for diamond detectors [8], suggesting that for these current densities, some recombination occurs even when the carrier velocity is saturated.
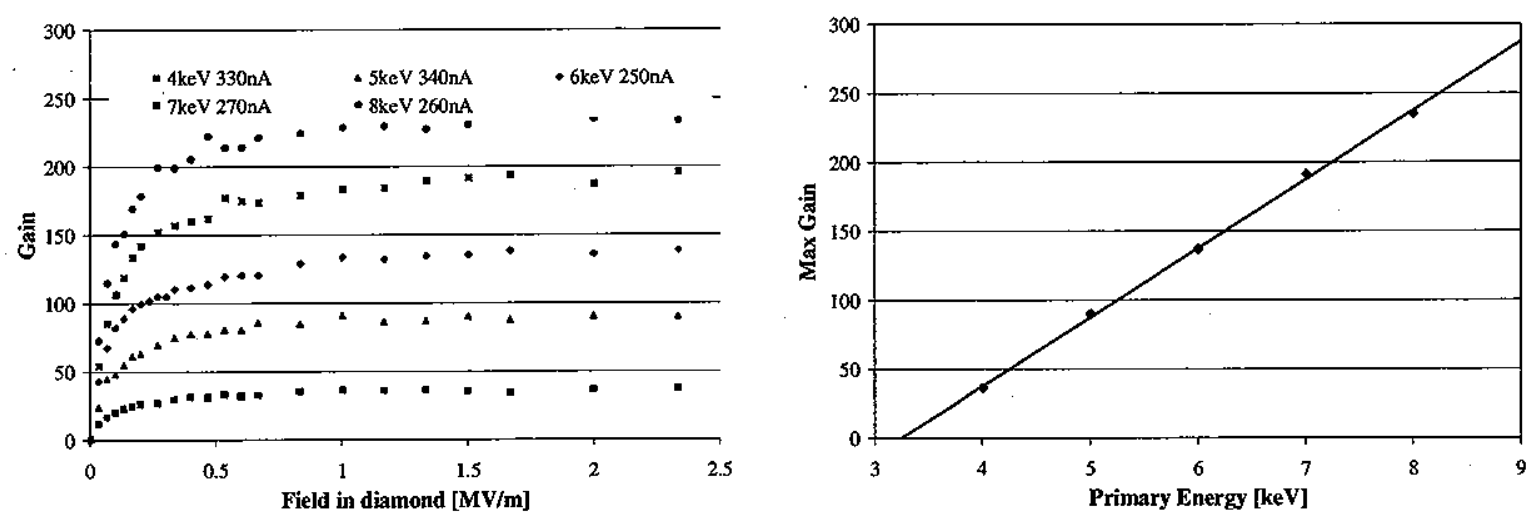

Figure 3. Left: gain for 4-8 keV primaries as a function of field in diamond in transmission mode; Right: saturated gain vs. primary energy

\section{Diamond Patterning}

As discussed above, for the amplifier to function in an RF cavity, the electrons must escape the diamond in a fraction of an RF cycle. For $0.7 \mathrm{GHz} \mathrm{RF}$, the escape time can be no more than $\sim 200$ ps. Electrons moving at the saturation velocity of $\sim 1.5 \times 10^{5} \mathrm{~m} / \mathrm{s}$ will cross $\sim 30 \mu \mathrm{m}$ of diamond in this time. Commercially available synthetic diamonds for this application are at least $100 \mu \mathrm{m}$ thick, thus the ability to thin and shape diamond in the emitting area will limit the frequency of an RF cavity that can utilize a DAP. Two methods of shaping diamond are being investigated - laser ablation and RIE.
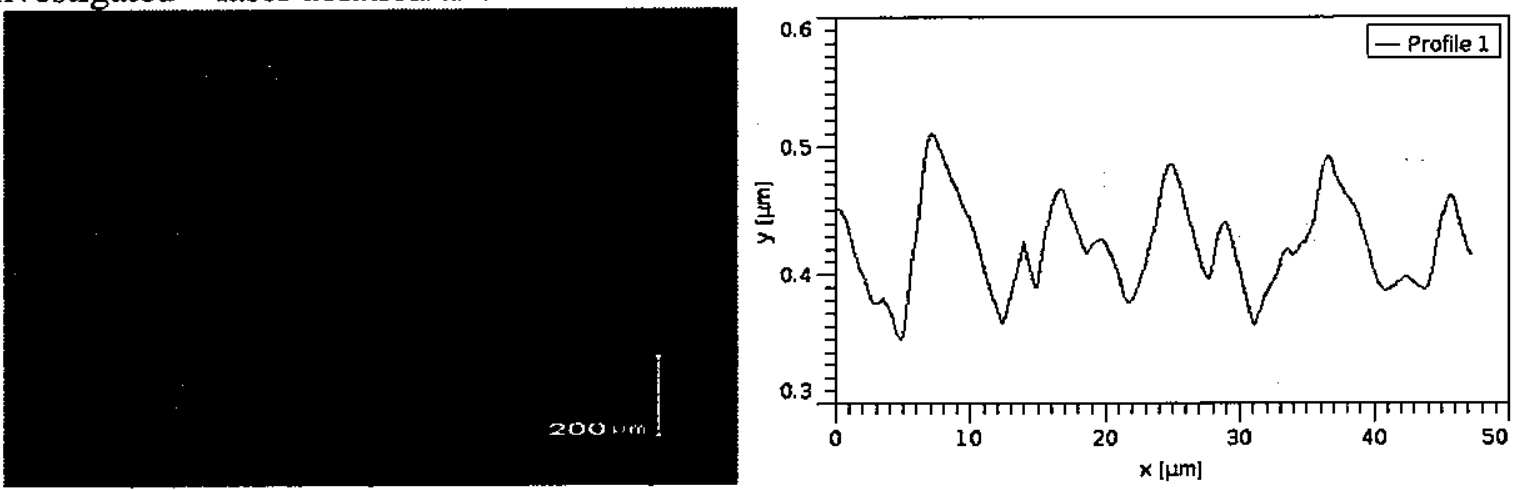

Figure 4. Left: laser ablated DD sample; Right: AFM profile of ablated area

Laser ablation rates of $\sim 17 \mu \mathrm{m} /$ minute have been achieved using a $248 \mathrm{~nm}$ excimer [9]. We have studied ablation of single and poly crystalline diamond at different wavelengths, pulse durations, intensities and in the presence of various oxygen concentrations [7]. We concluded 
that UV is more effective in ablating diamond while limiting cracking. Figure 4 shows a pattern cut into a DD sample with a $266 \mathrm{~nm}$ laser. The laser spot on the sample was Gaussian with a $\sim 30 \mu \mathrm{m}$ FWHM, a pulse duration of $20 \mathrm{ps}$, a pulse energy of $100 \mu \mathrm{J}$ and a repetition rate of $10 \mathrm{~Hz}$. A raster scan was used to create a $1 \mathrm{mmx} 4 \mathrm{~mm}$ line, with line spacing of $10 \mu \mathrm{m}$. The pattern was run 4 times, with a $5 \mu \mathrm{m}$ offset in the starting position. The total scan time was $10 \mathrm{hrs}$, and the depth of the line below the surface is $\sim 2 \mu \mathrm{m}$. The AFM profile (Fig. 4, right) shows the expected $5 \mu \mathrm{m}$ spacing between adjacent lines. The surface is smooth (on the $100 \mathrm{~nm}$ scale) apart from the structure caused by the Gaussian laser profile, indicating that with beam shaping and a larger laser spot, sufficient spatial uniformity should be achievable.

Although laser ablation looks promising to accomplish the majority of the desired patterning, it has some detrimental effects on the diamond surface. Amorphous carbon forms on the diamond surface (removable by ozone cleaning [7]). For polycrystalline material, some cracking is observed along grain boundaries. X-ray diffraction on the DD sample shows significant broadening of the 100 Bragg peak caused by laser ablation, indicating strain in the diamond. For these reasons, we are investigating RIE as a following step to laser ablation. RIE does not increase the stress in the diamond, and a $10 \mu \mathrm{m}$ RIE step following laser ablation significantly reduces the residual strain caused by the laser. We use an Oxford ICP (Inductively Coupled Plasma) RIE system, with a mixture of $\mathrm{O}_{2}$ and Ar gases with a 850W PICP power level, $15 \mathrm{~W}$ of $\mathrm{P}_{\mathrm{RF}}$, typically with a $50 \mathrm{sccm}$ flow of $\mathrm{O}_{2}$ and an $8 \mathrm{sccm}$ flow of Ar. Etch rates are routinely between $65-80 \mathrm{~nm} / \mathrm{min}$. The difference in the surface quality of OD and ED samples after RIE is seen in figure 5. These diamonds differ in the concentration of impurities and in the thermal conductivity (1100W/mK for OD and $1800 \mathrm{~W} / \mathrm{mK}$ for $\mathrm{ED})$.
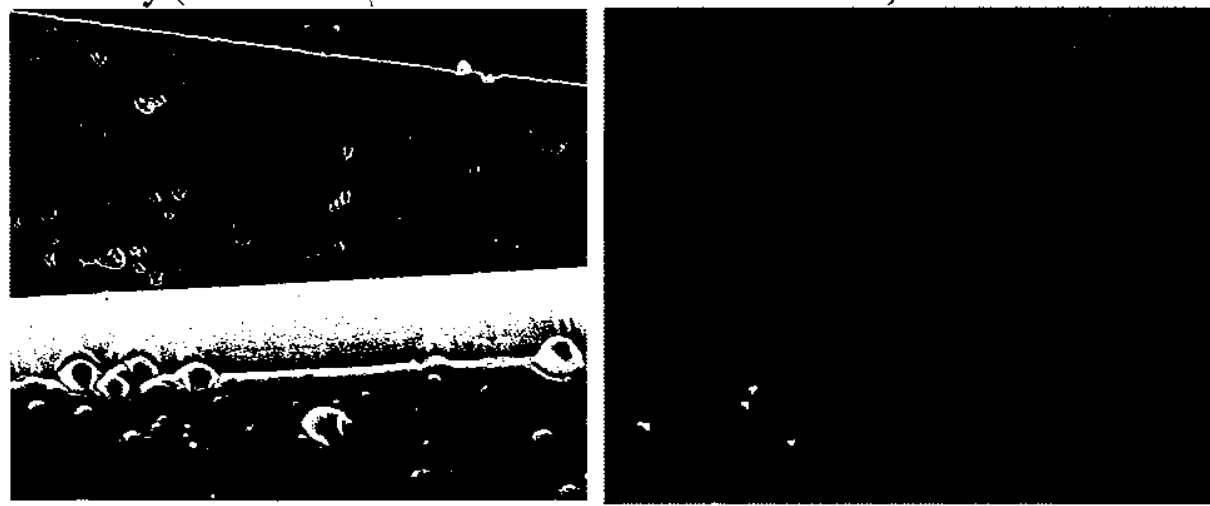

Figure 5. Left: RIE of OD sample; Right: RIE of ED sample (scale is common to both panels The OD sample (Fig. 5, left) clearly displays surface roughness on the scale of several microns, while the ED sample (Fig. 5, right) surface is apparently smooth, with only a few $<1 \mu \mathrm{m}$ imperfections. For the DAP application, a surface roughness of $\leq 100 \mathrm{~nm}$ is required to avoid electron bunch spreading. RIE of the ED sample achieves an acceptable surface.

\section{Braze and Metallization}

A metallization layer is required to provide charge resupply to the diamond. This layer must have a good conductivity, yet be thin enough to minimize energy loss of the primary electrons. A braze step is necessary to couple the metallized side of the diamond to the RF cavity. The thermal stress involved in the braze process is a concern, as the braze is a high temperature $(>1100 \mathrm{~K})$ eutectic alloy while the operating cathode will be at cryogenic temperatures $(<100 \mathrm{~K})$, resulting in a thermal cycle greater than $1000 \mathrm{~K}$. The braze of diamond to copper using TiCuSil (Wezgo) was demonstrated in a nTorr scale vacuum furnace. We found it necessary to decrease the thickness of the copper washer to reduce the tensile moment due to 
differential thermal expansion. A washer of thickness $>200 \mu \mathrm{m}$ exerted a high enough tensile moment to crack the diamond wafer on the inner diameter during cooldown. Figure 6 (left) shows a $100 \mu \mathrm{m}$ thick copper washer brazed to a $350 \mu \mathrm{m}$ thick OD sample. Finite element analysis has led to a braze joint design which will reduce the stress in diamond (Fig. 6, right).

The metallization of the diamond will follow the braze to avoid migration of $\mathrm{Ti}$ through the Pt capping layer [10] and subsequent Ti oxidation. To enable carbide formation and thus ohmic contacts to the diamond, we anneal Ti/Pt films in an inert He atmosphere rapidly $\left(3^{\circ} \mathrm{C} / \mathrm{s}\right)$ with an equally rapid cooldown [11]. We are currently investigating different carbide forming metals, such as Mo and $\mathrm{Nb}$, for establishing ohmic contact to diamond.
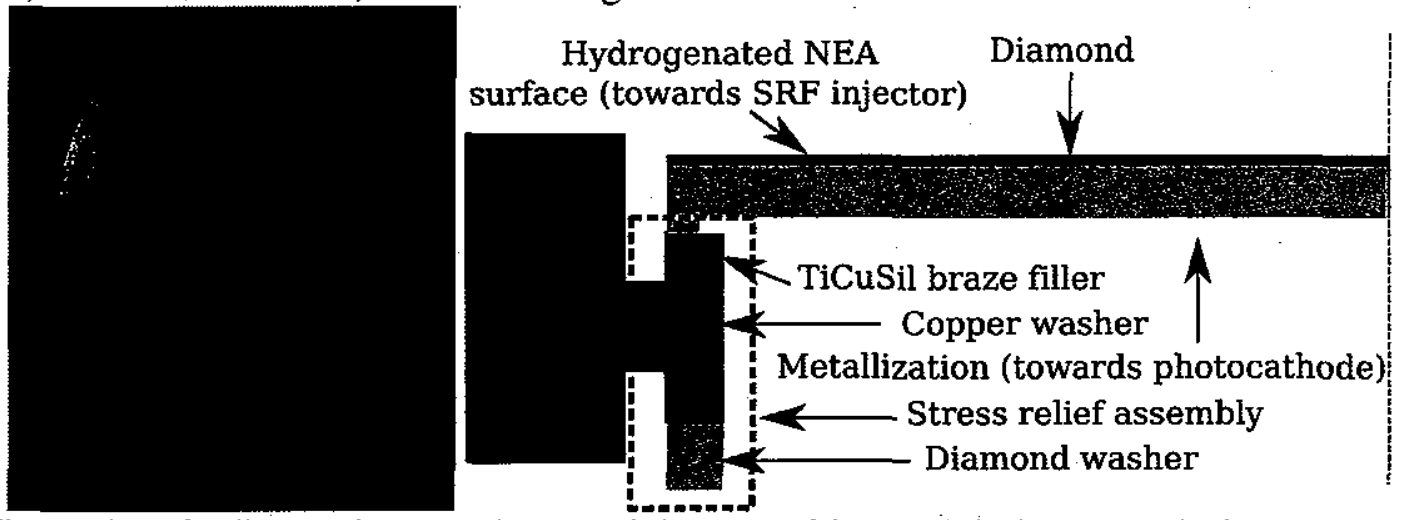

Figure 6. Left: diamond-copper braze; Right: assembly to minimize stress (axisymmetric)

\section{ACKNOWLEDGMENTS}

We would like to thank J. Walsh, B. Smith and D. Pate for expert technical assistance, R. Hemely's group at the Carnegie Institute of Washington for PL measurements, and J. Warren for SEM pictures. Several beamlines at the NSLS were instrumental to this work: X6B (E. Dimasi), X16C (P. Stephens, SUNYSB), X20C (J. Jordan-Sweet, IBM), U2A (Z. Liu, COMPRES, NSF EAR 01-35554) and U2B (CWRU, NIBIB, P41-EB-01979). This manuscript has been authored by Brookhaven Science Associates, LLC under Contract No. DE-AC02-98CH10886 with the U.S. Department of Energy.

\section{REFERENCES}

1. Ben-Zvi I. et al, Nuc. Inst. and Meth. in Phys. Res. A532 177 (2004)

2. W. R. Flavell et al, J. Phys.: Condens. Matter 16 S2405 (2004)

3. J. Smedley et al, in Proc. of AAC06, AIP Conference Proceedings Volume 877, p 672 (2006)

4. J.E. Yater et al, J. Appl. Phys. 878103 (2000); J. Appl. Phys. 97093717 (2005)

5. T. Watanabi, T. Teraji and T. Ito, J. Appl. Phys. 954866 (2004)

6. D. A. Dimitrov et al, Proc. of PAC07, Albuquerque, NM, p.3555 (2007)

7. T. Rao et al, Proc. Workshop on Energy Recovery Linacs, Daresbury, UK (May 2007)

8. C. Bauer et al, Nuc. Inst. and Meth. in Phys. Res. A383 64-74 (1996)

9. George T., et al, Appl. Phys Lett. 62, 2880 (1993)

10.U. Schmida and H. Seidel, J. Vac. Sci. Technol. A 242139 (2006)

11.W. P. Leroy, et al, J. Appl. Phys. 101, 053714 (2007) 